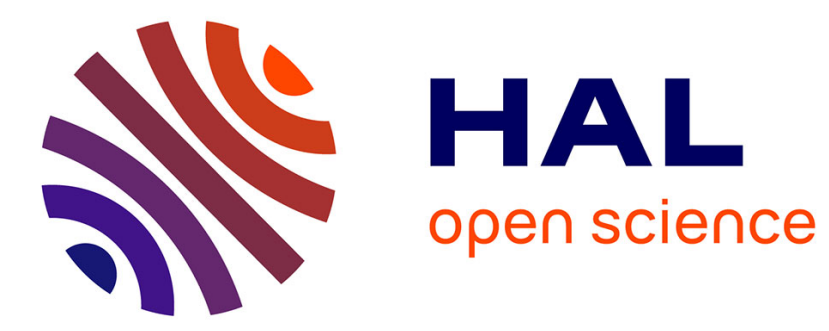

\title{
U2RV: UAV-assisted reactive routing protocol for VANETs
}

Omar Sami Oubbati, Omar Sami Oubbati, Noureddine Chaib, Abderrahmane Lakas, Salim Bitam, Pascal Lorenz

\section{- To cite this version:}

Omar Sami Oubbati, Omar Sami Oubbati, Noureddine Chaib, Abderrahmane Lakas, Salim Bitam, et al.. U2RV: UAV-assisted reactive routing protocol for VANETs. 2019, 10.1002/dac.4104 . hal$02301888 \mathrm{v} 2$

\section{HAL Id: hal-02301888 \\ https://hal.science/hal-02301888v2}

Submitted on 7 Oct 2019

HAL is a multi-disciplinary open access archive for the deposit and dissemination of scientific research documents, whether they are published or not. The documents may come from teaching and research institutions in France or abroad, or from public or private research centers.
L'archive ouverte pluridisciplinaire HAL, est destinée au dépôt et à la diffusion de documents scientifiques de niveau recherche, publiés ou non, émanant des établissements d'enseignement et de recherche français ou étrangers, des laboratoires publics ou privés. 


\title{
U2RV: UAV-Assisted Reactive Routing Protocol for VANETs
}

\author{
Omar Sami Oubbati, Member, IEEE, Noureddine Chaib, Senior Member, IEEE, \\ Abderrahmane Lakas, Member, IEEE, Salim Bitam, Member, IEEE, \\ Pascal Lorenz, Senior Member, IEEE
}

\begin{abstract}
When it comes to keeping the data routing robust and effective in Vehicular Ad hoc Networks (VANETs), stable and durable connectivity constitutes the keystone to ensure successful point-to-point communication. Since VANETs can comprise all kinds of mobile vehicles moving and changing direction frequently, this may result in frequent link failures and network partitions. Moreover, when VANETs are deployed in a city environment, another problem arises, that is, the existing obstructions (e.g., buildings, trees, hoppers, etc.) preventing the lineof-sight between vehicles, thus degrading wireless transmissions. Therefore, it is more complicated to design a routing technique that adapts to frequent changes in the topology. In order to settle all these problems, in this work, we design a flooding scheme that automatically reacts at each topology variation while overcoming the present obstacles while exchanging data in ad hoc mode with drones that are commonly called Unmanned Aerial Vehicles (UAVs). Also, the aim of this work is to explore well-regulated routing paths providing a long lifetime connectivity based on the amount of traffic and the expiration time of each discovered path, respectively. A set of experiments is carried out using simulation, and the outcomes are confronted with similar protocols based on a couple of metrics. The results clearly show that the assistance of UAVs to vehicles is capable to provide high delivery ratios and low delivery delays while efficiently extending the network connectivity.
\end{abstract}

Index Terms-VANET, UAV, Routing, Connectivity, Simulation.

\section{INTRODUCTION}

Since the appearance of wireless interfaces, wired connections in certain networks are surpassed in order to provide flexibility of movements to the nodes while adopting this kind of interfaces [1]. We consider that a network is mobile when the movements of nodes allow to establish new wireless connections and disconnections (i.e., appearance and disappearance of links). Called Mobile Ad hoc Network (MANET), is generally composed of self-organized mobile nodes characterized by their unstable behavior (i.e., random mobility), which can cause sometimes packet losses [2]. This kind of network has given birth to two popular types of networks (c.f., Fig. 1), called Vehicular and Flying Ad hoc Networks (i.e., VANETs

O. S. Oubbati \& N. Chaib are with the Computer Science and mathematics Laboratory, University of Laghouat, BP 37G, Ghardaïa Road, Laghouat 03000, Algeria. E-mail: \{s.oubbati, n.chaib\}@lagh-univ.dz.

A. Lakas is with College of Information Technology, United Arab Emirates University, United Arab Emirates. E-mail: alakas@uaeu.ac.ae.

S. Bitam is with LESIA Laboratory Department of Computer Science, University of Biskra, Algeria. E-mail: s.bitam@univ-biskra.dz.

P. Lorenz is with University of Haute Alsace, France.

E-mail: pascal.lorenz@uha.fr. and FANETs, respectively). Both networks are specified based on both the type of their mobile nodes and the environment where they are deployed. Moreover, each kind of networks adopts its own communication architecture in order to organize the exchanging of packets. Consequently, some techniques are inherited from MANETs to support data exchange between the nodes in these networks. Nevertheless, these techniques need to be adapted to the unique characteristics of such networks.

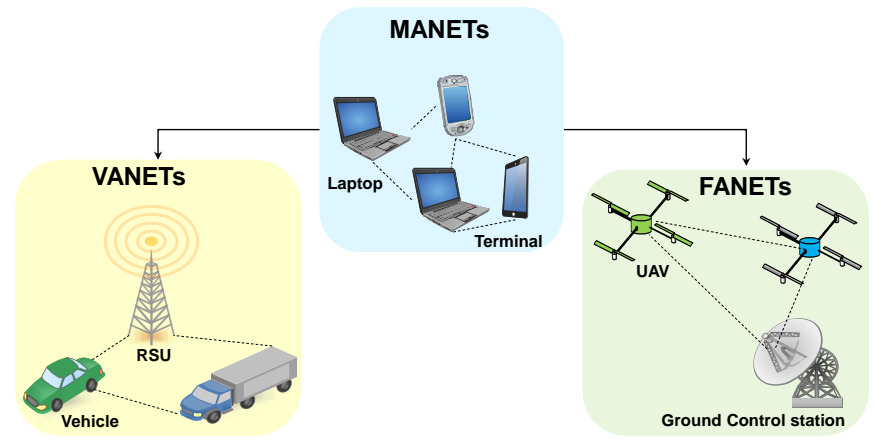

Fig. 1: MANETs subclasses.

Two sorts of wireless communications are used by VANETs, Vehicle-to-Vehicle (V2V) and Vehicle-to-RoadSide-Unit (V2R) [3], which have participated in the emergence of numerous useful road-safety and comfort applications (e.g., crashes' avoidance, Internet access, Weather information, etc.). The majority of these infotainment applications have a basic need in terms of throughput and data delivery delay [4]. However, these requirements are not simple to be guaranteed, especially in urban environments. For example, at a certain time, the direct communication link between two vehicles, or between vehicles and Road-Side-Units (RSUs) cannot be established due to existing obstructions. This can sometimes prevent the line-of-sight (LoS) between the communicating nodes and can distort the radio signals as well [5]. In addition, the highly dynamic mobility of vehicles caused by the sudden and frequent change of their directions can also disturb the already established wireless links resulting in many disconnections [6]. All these constraints have to be avoided by selecting an efficient way to ensure data delivery with lower packet losses. Exchanging messages between the road entities is essential, and especially when an accident occurs. In this case, vehicles have to share this information with neighboring vehicles, and if possible with security services. Sending this kind of information requires sometimes direct 
access to the Internet in order to share the exact location and other useful information about the crash [7]. This has to be done efficiently without packet losses because it is a question of human lives. Internet access in VANETs is usually insured by RSUs that are placed along the roads operating as gateways [8]. When vehicles want continuous access to the Internet, data packets or messages need to be sent directly to existing RSUs in range. However, this possibility is not always available, particularly when vehicles circulate in urban areas. Several constraints are distinguished in such environments, such as the limited number of RSUs, the high mobility of vehicles, the existing obstructions, etc. These issues can be addressed using efficient multi-hop communications strategies between vehicles to reach the target destinations (e.g., gateways).

An important number of multi-hop communications strategies (i.e., routing techniques) dedicated to urban VANETs have been proposed, which can be grouped into three categories: (i) Greedy-based routing, (ii) Delay tolerant-based routing, and (iii) Reactive-based routing. The first category is characterized by choosing the shortest routing path between a pair of communicating vehicles. Several studies [9]-[11] in this category have shown many weaknesses regarding the aforementioned issues, particularly the high mobility of the nodes since this category requires highly dense networks and preferably with low mobility. The second category is distinguished by making intersections the only landmark for routing decisions. In this category, the store-carry and forward is the most common technique, which is used when there are no connected paths to the target destinations. Several other studies [12]-[14] have also shown many issues, such as the high delays caused by the overuse of the store-carry and forward when the network is fragmented and suffers from frequent disconnections. The last category is mainly based on the flooding process, which is responsible for discovering all available paths to destination nodes. Numerous reactive-based routing protocols [15]-[17] have suffered from three important issues (i) the data congestion due to the additional packets used in the flooding process, (ii) the excessive overhead due to the dynamic size of exchanged packets, and (iii) the high delays caused by the discovery process, and especially when the network is intermittently connected. As a result, most of the protocols belonging to these categories focus only on studying the mobility of existing vehicles on the roads, which constitutes the major way of forwarding data packets. Moreover, most of the issues distinguished in the majority of protocols are mainly caused by the variation of the vehicle density.

Besides vehicles and RSUs, several other categories of mobile nodes can be distinguished in urban environments, such as trains, bicycles, and even pedestrians that can all be considered as relays (forwarders) to assist the data delivery in VANETs [18]. However, sometimes the movements of these nodes are localized in places very far from the location of vehicles. Hence, their inability to participate in the data delivery in a traditional VANET. In the last decade, a new category of aerial mobile nodes has been emerged called pilotless flying objects or Unmanned Aerial Vehicles (UAVs). The number of UAVs has been increased significantly, and most of them fly over urban roads. Consequently, UAVs can be considered as the most suitable solution to assist vehicles located on the ground during the routing process [19]. Moreover, UAVs can communicate in an ad hoc fashion through heterogeneous wireless communications with existing VANETs. Like vehicles, UAVs can also establish a connection to the Internet using multihop communications through vehicles or UAVs and they can avoid obstacles on the ground by relaying messages in the sky. In order to make the data exchange between vehicles more stable and efficient with the assistance of UAVs, several routing protocols adopting this concept have been proposed across the literature [20]. However, several weak points have been distinguished in these protocols on different sides, such as the data delivery process, the obstacle's avoidance, and the recovery. The primary objective of this research is to resolve individually all these encountered issues, to provide reliable communication links, and to be softly adjusted to the highly dynamic topology of such networks.

Numerous contributions are performed in this work as follows:

- A detailed illustration of different kinds of routing protocols proposed in the last decade, which we believe are the most relevant to shape our proposed routing protocol. A series of comparisons based on various parameters with the discussed protocols have been carried out demonstrating the effectiveness of the UAVs' assistance to existing VANETs employed by our protocol.

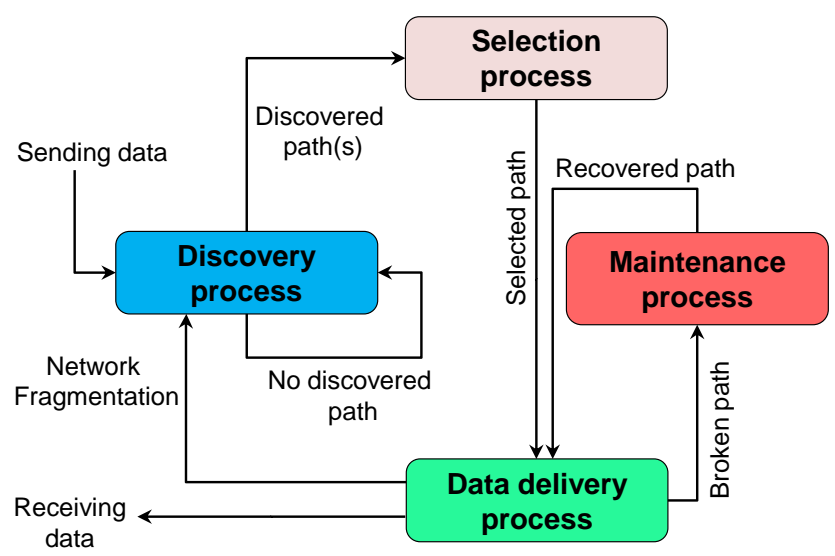

Fig. 2: Processes of U2RV.

- An extended version of our previous routing scheme [21] is described. As illustrated in Fig. 2, the scheme is based on four main processes that are significantly enhanced in order to be both smoothly adapted to the dynamic nature of VANETs and effective in terms of delivery ratio and delay: (i) Discovery process is intelligently used when there is a requirement to establish a routing path between a pair of source and destination nodes while predicting any link breakage before its occurrence, (ii) Selection process selects a suitable path based on a score using numerous metrics that are estimated gradually while establishing the path to the target destination, (ii) Data delivery process transits the data packet across the picked path to the target destination, and (iv) Maintenance 
process is deployed when there is an instability of the established paths.

- A deployment of this routing strategy is not feasible without discussing the different security issues. Therefore, a set of possible attacks over our approach has been studied along with the propositions of solutions. This study can be considered as future works and perspectives.

- A set of experiments has been performed under a simulation environment. The outcomes have been discussed and the effects of using UAVs as relays have been highlighted.

The remainder of this paper is outlined as follows. In Section II, we outline the relevant routing protocols that were used both to model our work and as comparison references during the experiment throughout this paper. In Section III, we detail the main processes of our protocol. Section IV presents the performance results of our protocol and evaluate them. Our work is concluded in Section V while studying the main threats against our routing scheme.

\section{RELATED WORK}

Routing is the keystone of any data exchange in VANETs. A lot of research work has been focused on proposing routing protocols in VANETs under different environments, such as urban and highway. However, certain issues always persist, such as the frequent disconnections of the network caused by several factors, such as the existing obstructions, the highly dynamic mobility of vehicles, and the high degrees of network overhead. To provide a long lifetime routing path despite the aforementioned challenges, routing based on flooding techniques is considered as the most common and suitable for VANETs [22]. In this category, discovered routing paths are established to the target destination during a given slot of time. In the following, a description is presented covering the most relevant routing for VANETs, which we believe are the most adequate to shape our own routing protocol. Since our protocol is assisted by UAVs in the sky, the knowledge of the various routing methods adopted for the data transition between UAVs is required. Therefore, a sample of routing protocols that are based on flooding dedicated to FANETs is also described.

\section{A. VANET routing protocols}

In the last decade, there have been several attempts to propose routing protocols for VANETs based on the flooding process into the network. As a result, several research works have been proposed on reactive-based routing.

Taleb et al. designed a routing protocol exploiting the moving directions of the vehicles so that to categorize them into different groups [23]. The routing stability is based on grouping vehicles using their velocity vectors and speed. The flooding is initialized by the source vehicle by broadcasting a route request (RREQ) packet in the network. After that, the routing decision is performed by the destination by selecting the most steady path. This path is integrated into the route reply (RREP) packet, and then, it will be sent back to the source. As an issue, this protocol does not consider the obstacles during the establishment of groups. To reduce the data loss in the video stream, the work in [24] adopts a tradeoff between a routing protocol and redundancy with retransmission of missing data.

Another different technique is designed and adopted by Zhaomin et al. [25]. In this approach, a metric has to be calculated named EDD (Expected Disconnection Degree), it represents a disconnection probability of a candidate routing path during a given period of time. The more reduced the value of EDD, the more steady is the path. The calculation of the EDD is done by combining several metrics that characterize the vehicle, such as the velocity, the position, and the trajectory. Consequently, the path in which vehicles have the same velocity vectors and speed is considered as the most stable, and therefore, more desirable to be selected as a routing path. When the shortest path to a destination needs to be calculated, the source initiates the flooding process. Concurrently, the EDD is progressively estimated at each hop and included in the RREQ. The destination selects the path with the best EDD value based on the intercepted RREQs. As a drawback, this scheme requires permanent connectivity of the network and can allow only a restricted time of network fragmentation.

A similar protocol is extended to take into consideration the vehicular density on the roads, was proposed in [26]. It further exploits a route discovery phase to both establish a routing path and get the position of the destination. During the selection phase, a weight is calculated for each discovered path (i.e., a succession of junctions) based on the vehicle density and delivery delay. The succession of intersections that obtains the best (highest) weight, it will be chosen to transmit the RREP packet back to the source based on the technique of greedy forwarding. However, this protocol neglects the accurate distribution of vehicles between two successive intersections, which can cause links breakage, even if this path contains a high number of vehicles.

Sun et al. developed a routing protocol that can be deployed over urban VANETs called GVGrid [27]. This protocol adopts a grid-based approach using a discovery phase to construct routing paths. The map is assumed to be initially divided into several grids. The RREQs are delivered along the roads through different grids in order to find routing paths composed of a minimum number of grids. Several useful information and parameters are recorded in the routing table, such as the source vehicle, the destined grid, and the grids sequence. If the routing path fails, GVGrid has to find an alternative vehicle in the next disconnected grid. In the case when fragmentation occurs, vehicles are not able to use the process of grids since there is no vehicle to be selected as a next hop. In addition, GVGrid supposes that the network is highly dense, which is not reasonable since, as widely known, the amount of traffic is dynamic and not constant during the whole day.

\section{B. FANET routing protocols}

Different routing schemes have been proposed for FANETs, in which most of them are based on a flooding scheme or a discovery process. This technique is suitable to support the high mobility of nodes. All those protocols concentrate on moving UAVs in the sky.

Shirani et al. adopted a reactive strategy exploiting the geographical locations of UAVs, which are stored in the 
TABLE I: VANETs and FANETs routing protocols features comparison.

\begin{tabular}{|c|c|c|c|c|c|c|c|c|}
\hline \multirow{3}{*}{ Features } & \multicolumn{4}{|c|}{ VANETs routing } & \multicolumn{3}{|c|}{ FANETs routing } & \multirow{3}{*}{ Our protocol } \\
\hline & ROMSGP & MURU & AGP & GvGrid & RGR & MUDOR & ARPAM & \\
\hline & Ref. [23] & Ref. [25] & Ref. [26] & Ref. [27] & Ref. [28] & Ref. [29] & Ref. [30] & \\
\hline Traffic density calculation & $\times$ & $\times$ & $\sqrt{ }$ & $\bar{x}$ & $x$ & $\bar{x}$ & $\bar{x}$ & $\sqrt{ }$ \\
\hline Connectivity estimation & $\sqrt{ }$ & $\sqrt{ }$ & $x$ & $x$ & $\times$ & $\sqrt{ }$ & $x$ & $\sqrt{ }$ \\
\hline Distribution of nodes & $x$ & $x$ & $\times$ & $\bar{x}$ & $\times$ & $x$ & $\times$ & $\sqrt{ }$ \\
\hline Obstruction considerations & $\sqrt{ }$ & $\times$ & $\sqrt{ }$ & $\sqrt{ }$ & $\times$ & $\times$ & $x$ & $\sqrt{ }$ \\
\hline UAV assistance & $\times$ & $\times$ & $x$ & $x$ & $\times$ & $\times$ & $x$ & $\sqrt{ }$ \\
\hline Path expiration & $\sqrt{ }$ & $\sqrt{ }$ & $\times$ & $x$ & $x$ & $\sqrt{ }$ & $\sqrt{ }$ & $\sqrt{ }$ \\
\hline Maintenance process & $\sqrt{ }$ & $\sqrt{ }$ & $\sqrt{ }$ & $\sqrt{ }$ & $\bar{x}$ & $\sqrt{ }$ & $\sqrt{ }$ & $\sqrt{ }$ \\
\hline Target destination (receiver) & $U A V$ & $U A V$ & $U A V$ & $U A V$ & $U A V$ & $U A V$ & $U A V$ & $U A V / R S U$ \\
\hline
\end{tabular}

routing tables of intermediate UAVs during the transition of the RREP packet [28]. If the reactive routing fails, RGR can suffer from the local optimum problem when the GGF takes place.

The authors of [29] proposed Multipath Doppler Routing (MUDOR) designed for FANETs. MUDOR selects the longest lifetime paths, which can be considered as the most stable ones. MUDOR uses the discovery phase towards the target destination. Initially, each UAV intercepts the disseminated RREQ, it includes the measured value of the Doppler from the previous node and rebroadcasts it. This can determine the identifiers of the communicating aerial vehicles and their relative velocity. After that, MUDOR takes into account all the calculated Doppler values to select the path that has the longest lifetime. The discovery phase can cause the problem of congestion, which can also result in additional delays.

Iordanakis et al. designed a position-based routing protocol [30]. This is inspired by the functionality of AODV [31], and consequently, it can be reactive. The flooding process is used by the source UAV using RREQ packets, which include two additional information: (i) the position and (ii) the velocity vector of the source. These parameters can both provide the transition distance of the packet and determine the current location of the source node. A routing decision is performed by unicastly sending an RREP packet back to the source. This protocol can be reactive or proactive, which can be considered as its main drawback regarding the problem of congestion.

Our routing protocol proposed in this paper complements all the aforementioned related works by adopting a new concept of hybrid communications between vehicles and UAVs, aiming to improve the routing process regarding several sides. This opens up a promising research area by adopting this new kind of wireless communication. Table I presents a comparative study based on different features of the previously described protocols for VANETs and FANETs with the features adopted by our proposed protocol.

\section{U2RV: UAV-Assisted REACTIVE ROUTING PROTOCOL FOR VANETS}

After describing several proposed works in the literature and mentioning their shortcomings, a detailed depiction of U2RV and its four main processes are presented in this section.

- Discovery process: it is used to explore robust paths between the communicating nodes (i.e., whether they are gateways, vehicles, or UAVs). The established paths can comprise all kinds of nodes that are used as relays.
- Selection process: the destination calculates a couple of metrics, such as the density, the connectivity degree, the delivery delay, and the expiration time of each discovered path. All these criteria are combined to calculate a score for each discovered path and to select the path with the highest score for the data delivery.

- Data delivery process: data packets make their transition along their respective selected routing paths.

- Maintenance process: a permanent connectivity of a selected routing path is not possible due to the dynamic topology of the network. Consequently, always finding an alternative is a paramount condition.

Before detailing the functionality of our approach, an initial description of both the system model of U2RV and the communication way between nodes is required. Then, the main processes of this protocol are detailed.

\section{A. System Model}

Let us detail the system model and the different assumptions taken into account by U2RV. Consider a VANET deployed in a geographically limited urban city in which a set of UAVs, hovering in the sky, communicates and exchanges data with vehicles located on the ground. As depicted in Fig. 3, the network (i.e., the road segments and airspace) is assumed to be splitted into numbered static size zones (e.g., see Figs. 3(a) and 3(b), respectively). The size of these zones is defined based on the communication range of the nodes belonging to them. To have a deep understanding of our network division, several fixed zones are identified by a unique ID (c.f., Fig. 3(c)), since our routing process is not primarily based on the nodes, but it relies basically on the zones. This decomposition is crucial for the knowledge of important information, such as the amount of traffic, the accurate distribution of the nodes (i.e., UAVs and vehicles), and the destination's location. Both the identity and the geographical information of each zone are supposed to be known by all vehicles and UAVs using their included digital map. Each UAV or vehicle is able to determine its current geographical position based on the embedded GPS. Moreover, we suppose that there is no energy limitation for UAVs since they can be fitted with reloadable batteries that are permanently supplied through the resources of the UAV, such as gasoline, solar energy, etc [20].

It should be stressed that UAVs hover at a low-level flight that does not outstrip $\approx 1000 \mathrm{~m}$ during the flight in order to better serve vehicles on the ground. Moreover, vehicles, UAVs, and RSUs are uniformly distributed over the network. 


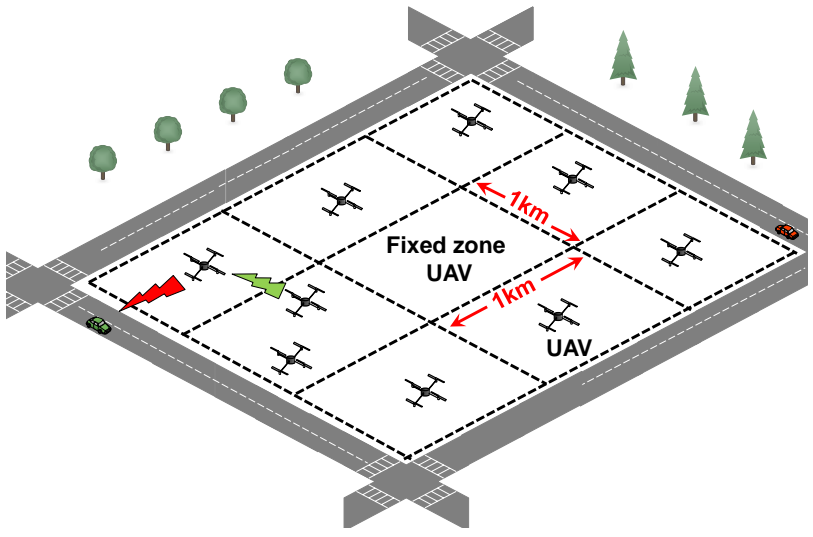

(a) Airspace divided into fixed zones.

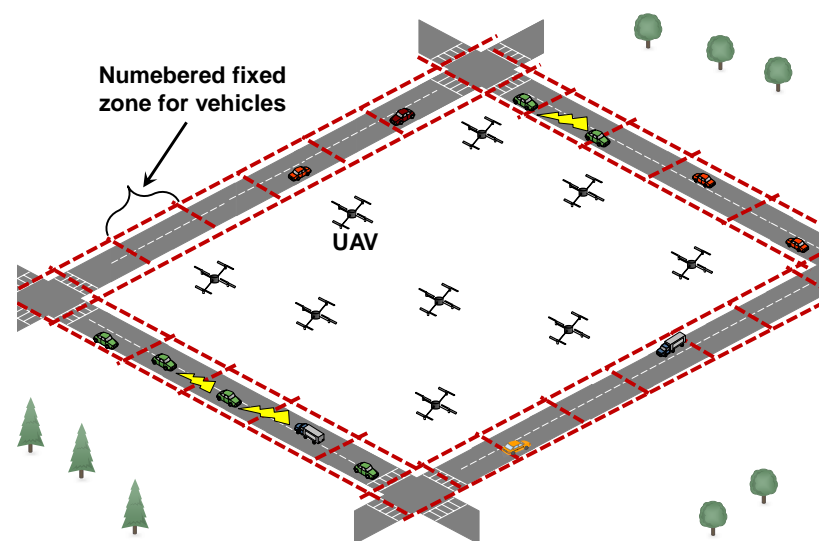

(b) Road segments divided into fixed zones.

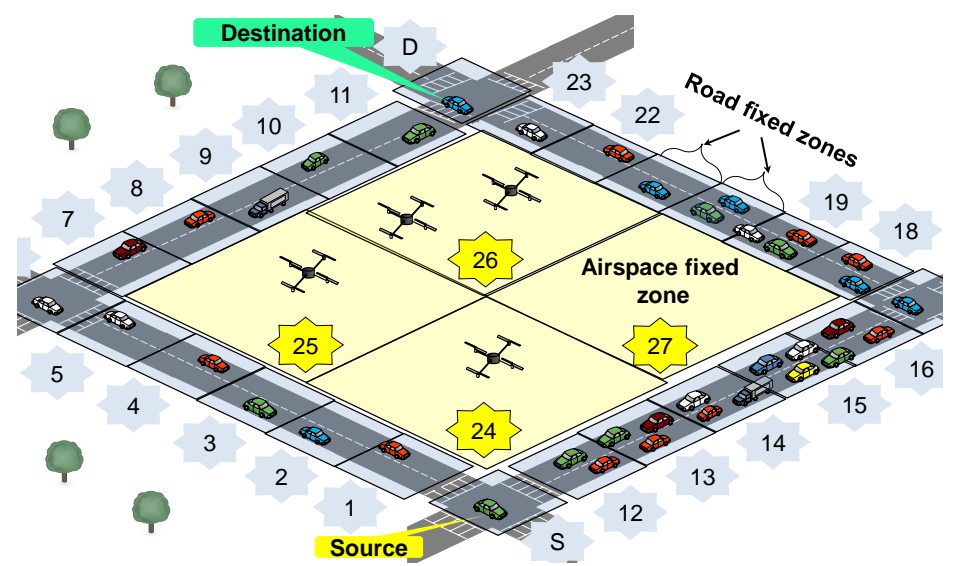

(c) Phase of the identification of fixed zones.

Fig. 3: Network organization.

They can communicate with each other based on incorporating wireless interfaces adopting the IEEE $802.11 \mathrm{p}$ standard, which can provide a high line-of-sight (LoS) probability [32]. As shown in Fig. 5, five types of wireless communications can be provided using this configuration:

1) Vehicle-to-Vehicle $(\mathbf{V} 2 \mathrm{~V})$ : vehicles on the ground within the LoS communication range of each other can communicate. In the case where there is an obstacle, the communication is not possible.

2) Vehicle-to-UAV (V2U): vehicles and UAVs can communicate with each other. Overall, UAVs in urban areas do not fly at high altitudes since most of their applications need to be done at low altitudes, such as in [33], or in our selected simulation scenario.

3) UAV-to-UAV (U2U): UAVs can exchange data between them if the distance separating them is no longer than their respective ranges.

4) Vehicle-to-RSU (V2R): in the majority of cases, this kind of communication is carried out in the case of applications requiring direct access to the content provided by the gateways (i.e., RSUs).

5) UAV-to-RSU (U2R): several UAV-based applications require Internet access for their right functionality. UAVs establish a connection with an RSU in range either to supply their current applications or to relay data packets from another node in the network.

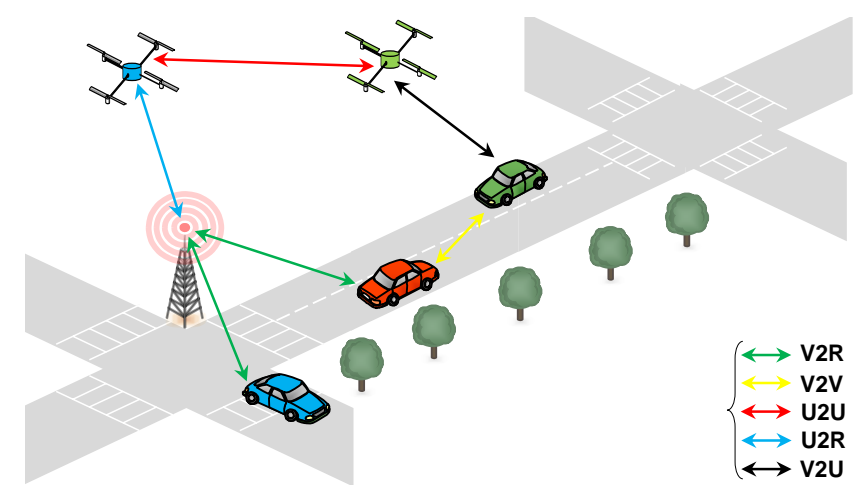

Fig. 5: Types of wireless communications.

\section{B. $U 2 R V$ packets format}

For a better understanding of the U2RV functioning, we provide details of the different packet formats used across the four processes of our protocol (c.f., Fig. 4). As depicted in Fig. 4(a), several fields characterize the RREQ packet. The RREQ ID field defines the communication's identifier between a unique pair of source and destination. The Delay field calculates the time required between the generation time 


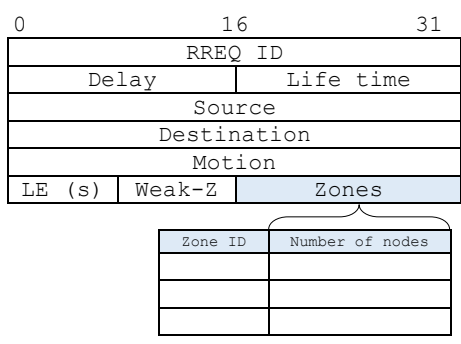

(a) RREQ packet format.

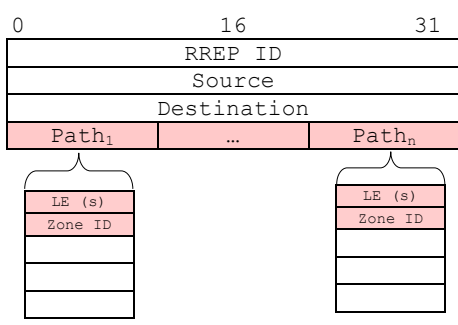

(b) RREP packet format.

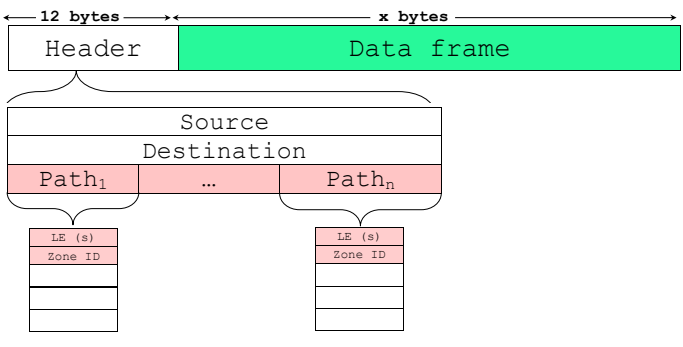

(c) Data packet format.

Fig. 4: U2RV packets format.

of the RREQ packet, and the reception time of the data packet. The Life time field represents the duration time of the flooding, which is initially set to a certain value. If Life time reaches its expiration, the RREQ will be dropped in order to limit the permanent dissemination over the whole network. All information about the Source and the Destination, such as their identifiers and locations are included into the RREQ packet. The Motion field is used to estimate the link lifetime between two neighbor nodes, where intermediate nodes modify it at each hop by adding their movements' information (i.e., position, velocity, and speed). The Weak $-Z$ field represents the zone where the links between its nodes are the weakest one in the discovered path.

The link expiration $L E$ of a given path is defined as the lowest duration of two given successive nodes belonging to this path to stay connected. $L E$ and Weak $-Z$ fields are exploited during the maintenance process to determine an alternative path when the selected path has been disconnected during the data transmission. Zones is a field that comprises a list of zones' identifiers with their respective densities, which is transited by the RREQ packet between the communicating nodes (i.e., Source and Destination). As illustrated in Fig. 4(b), the RREP packet format contains several fields. Certain fields are the same included in the RREQ packet, such as the information about the source and destination. The RREP ID field defines a unique communication session between a given source and destination. The discovered Paths in the form of zones' succession along with their expiration times are all included in the RREP packet. In Fig. 4(c), the data packet consists of two main fields: (i) a header of 12 bytes that contains similar fields as in the RREP packet, and (ii) a data frame restricted to $x$ bytes. These fields are exploited to deploy different techniques required for data delivery, such as the greedy forwarding and recovery techniques.

\section{Estimation of the link expiration}

In the case of willingness to send a data packet to a given node in the network, the source node generates an RREQ packet. Before proceeding to the flooding of this generated RREQ, the requesting node encloses its own mobility details (i.e., location, speed, and velocity), other information in other fields (as mentioned in the RREQ packet format description), and all into the RREQ packet. Initially, the expiration time of the path $L E$ is set to a maximum value. Then, the flooding process is started by the Source. When a forwarder intercepts the RREQ packet, it extracts the broadcaster mobility details and uses them with its own ones in order to calculate $L E$ of the current wireless link. The $L E$ field is modified on the new generated RREQ if the lifetime of the current wireless link is smaller than $L E$ already included.

As an illustration, we distinguish two possible scenarios (c.f., Fig. 6): (i) nodes moving in a two dimensional (2D) space (e.g., two UAVs with same altitudes or two vehicles) and (ii) nodes moving in a three dimensional (3D) space (e.g., two UAVs hovering with different altitudes or a UAV and vehicle). In the first scenario (c.f., Fig. 6(a)), let node n $_{1}$ and node 2 be two vehicles moving with limited speeds $V_{1}$ and $V_{2}, R$ is the LoS communication range of the two vehicles, $\left(X_{1}, Y_{1}\right)$ and $\left(X_{1}, Y_{1}\right)$ their respective geographical coordinates, and $\theta_{A}$ and $\theta_{A}$ their velocity angles (see Fig. 6(a)). Based on all this information, $L E$ of the link between node 1 and node $_{2}$ is calculated based on the same equation used in [34] as follows:

$$
L E=\frac{-(p s+q t)+\sqrt{\left(p^{2}+q^{2}\right) R^{2}-(p t-s q)^{2}}}{p^{2}+q^{2}}
$$

where,

$$
\begin{aligned}
p & =V_{1} \cos \theta_{1}-V_{2} \cos \theta_{2} \\
q & =V_{1} \sin \theta_{1}-V_{2} \sin \theta_{2} \\
s & =X_{1}-X_{2} \\
t & =Y_{1}-Y_{2}
\end{aligned}
$$

In the second scenario, let two UAVs (or a vehicle and $\mathrm{UAV})$, node $_{1}$ and node $_{2}$, are within each other's transmission range $R$ (c.f., Figs. $6(\mathrm{~b})$ and $6(\mathrm{c}))$. Let $\left(X_{1}, Y_{1}, Z_{1}\right)$ and $\left(X_{2}\right.$, $\left.Y_{2}, Z_{2}\right)$, be the coordinates of the two nodes, respectively. $V_{1}$ and $V_{2}$ are their moving speeds. $\theta_{1}, \phi_{1}$ and $\theta_{2}, \phi_{2}$ are their respective velocity angles. $L E$ of the link between these two nodes can be calculated based on the same method in [35] as follows:

$$
L E=\frac{-(p t+q u+s v)}{t^{2}+u^{2}+v^{2}}+z
$$




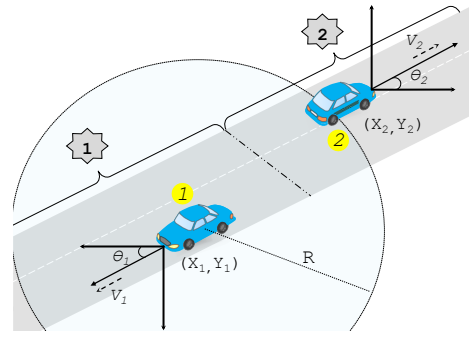

(a) Between vehicles.

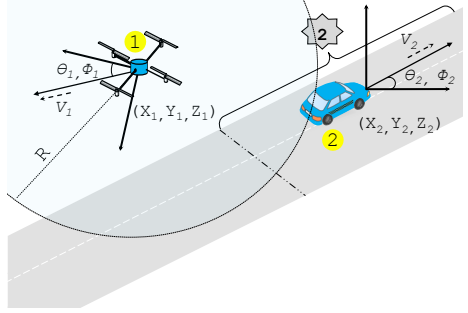

(b) Between vehicles and UAVs.

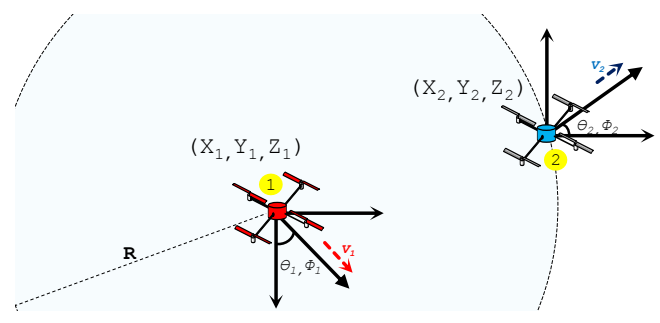

(c) Between UAVs.

Fig. 6: Link expiration calculation.

where,

$$
\begin{aligned}
p & =\left(X_{1}-X_{2}\right) \\
q & =\left(Y_{1}-Y_{2}\right) \\
s & =\left(Z_{1}-Z_{2}\right) \\
t & =\left(V_{1} \cos \theta_{1} \cos \phi_{1}-V_{2} \cos \theta_{2} \cos \phi_{2}\right) \\
u & =\left(V_{1} \sin \theta_{1} \cos \phi_{1}-V_{2} \sin \theta_{2} \cos \phi_{2}\right) \\
v & =\left(V_{1} \sin \phi_{1}-V_{2} \sin \phi_{2}\right) \\
z & =\frac{\sqrt{(p t+q u+s v)^{2}-\left(t^{2}+u^{2}+v^{2}\right)+\left(p^{2}+q^{2}+s^{2}-R^{2}\right)}}{t^{2}+u^{2}+v^{2}}
\end{aligned}
$$

\section{U2RV processes}

Let us consider Fig. 7 to illustrate the functioning details of $\mathrm{U} 2 \mathrm{RV}$ and their four main processes. The routing paths (i.e., succession of zones) are gradually discovered and built to the target destination using a flooding technique. In the subsequent sections, each process will be described in detail.
1) Discovery process: When there is a possible need to establish a connection with a particular node (i.e., fixed or mobile), a discovery process is initialized to get the maximum of routing paths towards the destination. In this process, the source node uses the flooding technique by broadcasting an RREQ packet across the network through all existing nodes. This broadcast allows the source vehicle to discover all existing paths in the form of zones' succession towards the target destination. Also, this process allows to getting the geographical position of the destination node and its corresponding zone as well. Concurrently, a set of metrics is gradually calculated, such as the amount of traffic, the real distribution of vehicles, $L E$, and the connectivity degree of each discovered path. Robust and well-stable paths are ideally desired by $\mathrm{U} 2 \mathrm{RV}$ in order to be used as data relaying paths supporting both the data transmission and the recovery. Each path is represented by a succession of zones, which can be stored in the RREQ packet. The latter is used by the target

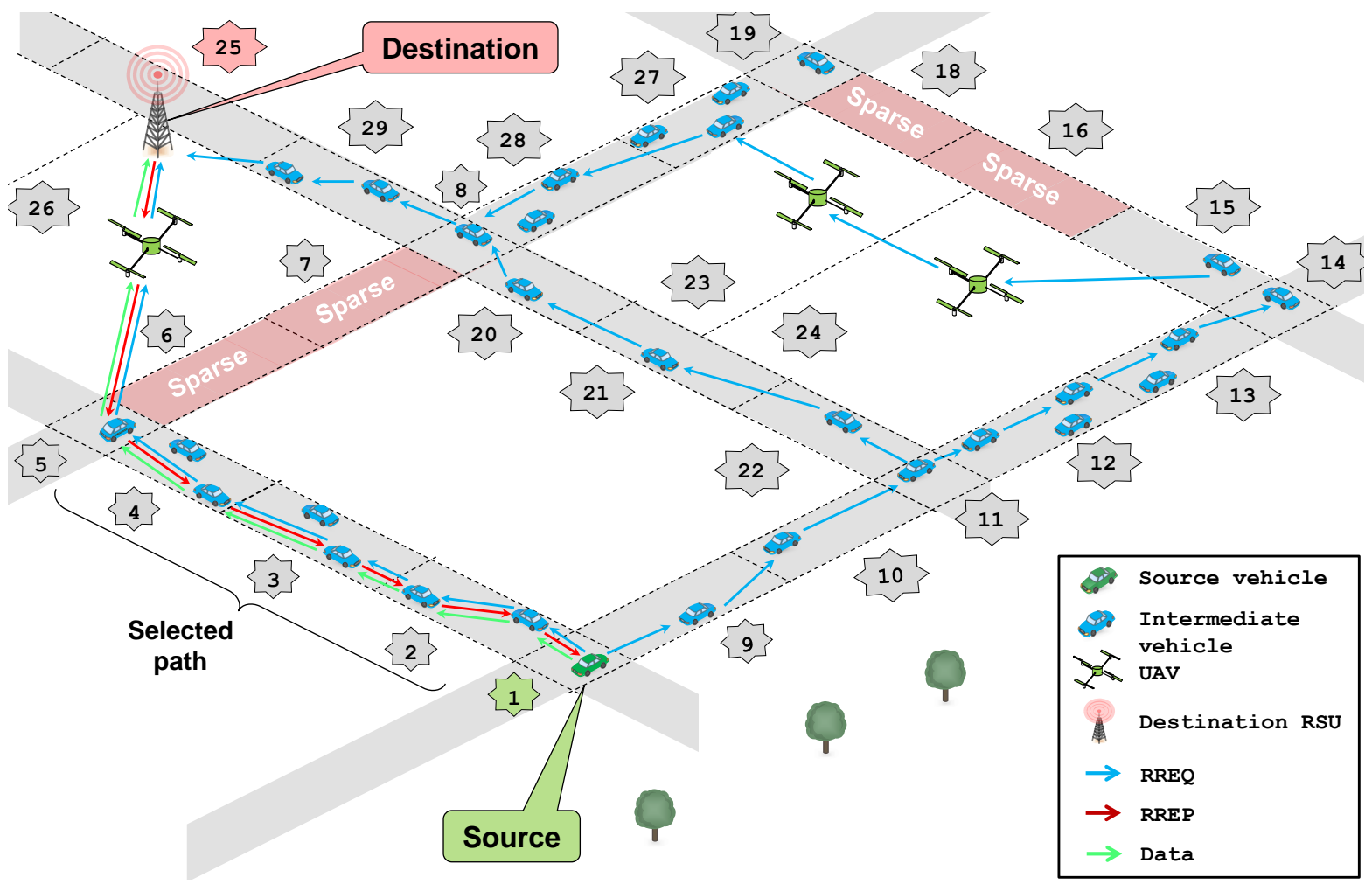

Fig. 7: Principle of functioning of U2RV. 
destination and intermediate nodes to accurately differentiate between the discovered paths.

As shown in Fig. 7, let vehicle $e_{1}$ be a source localized at Zone $_{1}$. If vehicle 1 wants to establish a routing path with a fixed destination gateway (RSU) located at Zone 25 , it starts the dissemination of an RREQ packet over the network to plot all routing possibilities towards the destination as well as to get both its geographical information and the zone ID of its location. The broadcast storm is significantly reduced thanks to the unique RREQ ID included in the broadcasted RREQs, thus dropping all intercepted RREQs already disseminated. The source node starts the broadcast of the RREQ in all directions (i.e., broadcasts an RREQ to its two neighboring direct zones Zone $_{2}$ and Zone 9 ). Based on the next zones, two different paths towards the destination are defined, since they comprise at least one different zone. Each transited zone along with its density is added to the RREQ packet. All this information will be eventually used during the data delivery. It is worthy to note that vehicles periodically exchange Hello packets between each other in order to be able to estimate the density in each zone. There is a case where a vehicle receives two RREQs coming from different paths, which are known from their Zones fields. We take as an example the vehicle located at the zone Zones, which carries out two different broadcasts to the only next Zone 26 . In this case, the target destination will receive two different RREQs, with two different Zones, and representing two different paths. As a result, the number of paths can be increased at crucial points, such as intersections and UAVs, where a forwarder node has many possibilities to broadcast the RREQ to different next zones (see Fig. 8 by zooming in Fig. 7). The importance of UAVs, as well as intersections, is increasing the number of alternative solutions. These next zones are used when the network becomes sparsely connected.

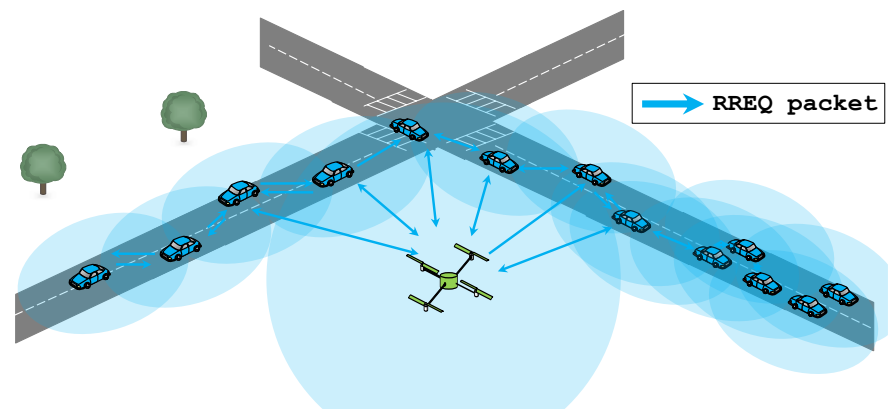

Fig. 8: RREQ flooding.

In the end, the target destination starts a timer as soon as it gets the first RREQ packet. This allows waiting an interval of time to intercept the maximum of RREQs, which correspond to existing paths to the source node. The waiting time is set to $50 \mathrm{~ms}$, in which all nodes do not change their positions a lot. These modifications of positions are considered as a minor variation compared to their limited speeds in urban areas. However, beyond this interval of time, all intercepted RREQs will be neglected, and the dissemination is considered to be achieved. In this case, the destination gateway located at
Zone $_{25}$ has to take a routing decision by selecting a suitable path for the data delivery.

2) Selection process: The destination makes a selection only in the case where there are different discovered paths where a decision is performed to select the most stable path between the communicating nodes. A score is calculated by the destination for each intercepted succession of zones based on several metrics already included in each received RREQ packet. A succession of zones with a low score is not suitable because they are the most confronted with the problem of disconnection. Once a succession of zones is selected, an RREP packet is generated and transmitted back to the source node through this succession. The RREP includes all necessary information about the selected path in order to be exploited during the maintenance process.

From the example of Fig. 7, several paths are received by the destination. Different parameters are calculated during the transition of the RREQ except for the final score, which is calculated by the target destination by combining the received metric values. The destination has a global vision of accurate metrics of three different paths. These crucial metrics represent the degree of path robustness. The higher value of the Score means the better robustness of the path. All necessary metrics of each path are described in TABLE II.

The calculation of the metrics related to the density, such as the real distribution of nodes $\sigma$, the average number of nodes $\mu$, and the total number of nodes $N$ (Zones) in a given path is carried out as follows:

$$
N(\text { Zones })=\sum_{i=1}^{\mid \text {Zones } \mid} N\left(\text { Zone }_{i}\right)
$$

where $\mid$ Zones $\mid$ is the number of transited zones, which is continuously incremented, while the RREQ packet is in transition at different zones in the path. The increment is stopped by reaching the target destination. $N\left(\right.$ Zone $\left._{i}\right)$ defines the number of nodes that are present at a specific zone $Z_{o n e_{i}}$. The average number of nodes per zone $(\mu)$ is calculated as follows:

$$
\mu=\frac{N(\text { Zones })}{\mid \text { Zones } \mid}
$$

where $N($ Zones $)$ is the integral density the full Path $h_{i}$ The formal equation of the standard deviation of zone densities $\sigma$ can be presented as follows:

$$
\sigma=\sqrt{\frac{1}{\mid \text { Zones } \mid} \times \sum_{i=1}^{\mid \text {Zones } \mid}\left(N\left(\text { Zone }_{i}\right)-\mu\right)^{2}}
$$

A high value of $\sigma$ means that the nodes in the zones are broadly spread around the average and vice versa. Disconnection can be caused if we obtain a small value of $\sigma$, which corresponds to the unfair distribution of nodes at a specific $P a t h_{i}$. If we take into consideration all intercepted information and the aforementioned calculated metrics, a score can be calculated for each $\mathrm{Path}_{i}$ based on the following equation:

$$
\text { Score }=\left\lfloor\frac{L E}{\text { Delay }}\right\rfloor \times\left(\frac{N(\text { Zones })}{1+\sigma+N(U A V)}\right)
$$


For the sake of clarity, several remarks can be deducted from the equation (6) as follows:

- When $\left\lfloor\frac{L E}{\text { Delay }}\right\rfloor=0$, it means that the path will be disconnected more probably before a successful data transmission. However, if $\left\lfloor\frac{L E}{\text { Delay }}\right\rfloor>0$, it means that the path will be expired after at least a successful data transmission.

- Score grows only on the positive side and it has a proportional relationship with $N$ (Zones) and $L E$. These two metrics are crucial to determine the connectivity degree of a path.

- Score has a disproportional relationship with Delay, $N(U A V)$ (i.e., number of hops involving UAVs), and $\sigma$ ).

- A path with the highest score means that it is the most stable and durable to make more data transmissions.

When the destination selects the appropriate path (e.g., Path $_{1}$ in Fig. 7 and in TABLE II), the gateway generates an RREP packet that includes all the required information for the data delivery, such as the selected succession of zones and the other discovered paths. Then, it is unicastly sent back to vehicle $_{1}$ based on the technique of greedy forwarding along the selected path (c.f., Fig. 9 by zooming in Fig. 7). This packet contains all required information in order to be able to make the reverse path, and that even if the selected path will be disconnected at a certain moment. The RREP packet is sent unicastly to the next zone (i.e., Zone 26 in Fig. 7), which belongs to Path . At each hop, the RREP packet has to be checked before each transmission in order to be transmitted correctly through the selected path to vehicle . $_{\text {. }}$

At the reception of the RREP packet by vehicle $e_{1}$ located at

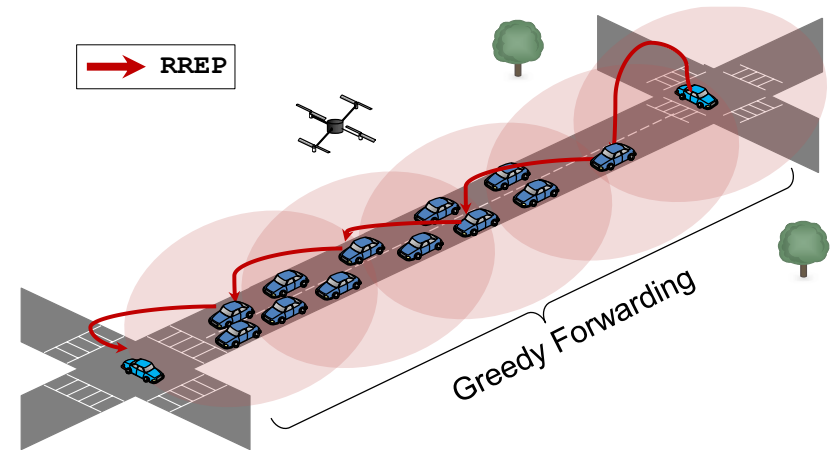

Fig. 9: RREP's unicast.

Zone $_{1}$, vehicle $_{1}$ starts the data transmission over the selected path (c.f., Fig. 7). For each data transmission, the data packet has to transit along each zone of the selected path. If a disconnection has occurred, there will be a possibility to find an alternative solution at each hop. Over 10 (s) of inactivity, the discovery process is a mandatory condition to make other data transmissions.

3) Data delivery process: For now, the data delivery is carried out using the greedy forwarding technique, and that at each time when the situation of the network allows it (c.f., Fig. 11 by zooming in Fig. 7). If this path will be disconnected at a certain time, a maintenance technique is used to recover the path in order to avoid re-initializing the discovery process. An essential condition for delivering data packets is intercepting the RREP packet by the sender. Once all information included in the RREP packet is merged with the data frame to be sent, the source node starts the data transmission along the selected path already transited by the RREP packet.

TABLE II: Discovered paths.

\begin{tabular}{|c|c|c|c|c|c|}
\hline \multicolumn{2}{|r|}{ Path $_{1}$} & \multicolumn{2}{|r|}{ Path $_{2}$} & \multicolumn{2}{|r|}{ Path $_{3}$} \\
\hline \multicolumn{2}{|c|}{ Zones $=7$} & \multicolumn{2}{|c|}{ Zones $=10$} & \multicolumn{2}{|c|}{ Zones $=15$} \\
\hline \multicolumn{2}{|c|}{$N($ Zones $)=10$} & \multicolumn{2}{|c|}{$N($ Zones $)=11$} & \multicolumn{2}{|c|}{$N($ Zones $)=23$} \\
\hline \multicolumn{2}{|c|}{$\sigma=0.53$} & \multicolumn{2}{|c|}{$\sigma=0.31$} & \multicolumn{2}{|c|}{$\sigma=0.74$} \\
\hline \multicolumn{2}{|c|}{ Delay $=1.2(s)$} & \multicolumn{2}{|c|}{ Delay $=2.5(s)$} & \multicolumn{2}{|c|}{ Delay $=4.5(s)$} \\
\hline \multicolumn{2}{|c|}{$L E=8(s)$} & \multicolumn{2}{|c|}{$L E=2.7(s)$} & \multicolumn{2}{|c|}{$L E=4,6(s)$} \\
\hline \multicolumn{2}{|c|}{ Weak $-Z=$ Zone $_{4}$} & \multicolumn{2}{|c|}{ Weak $-Z=$ Zone $_{21}$} & \multicolumn{2}{|c|}{ Weak $-Z=$ Zone $_{24}$} \\
\hline Zone & Number of nodes & Zone & Number of nodes & Zone & Number of nodes \\
\hline Zone $_{25}$ & $\begin{array}{l}1 \text { (Source) } \\
2 \\
2 \\
2 \\
1 \\
1 \text { (UAV) } \\
1 \text { (Destination) }\end{array}$ & $\begin{array}{l}\text { Zone }_{1} \\
\text { Zone }_{9} \\
\text { Zone }_{10} \\
\text { Zone }_{11} \\
\text { Zone }_{22} \\
\text { Zone }_{21} \\
\text { Zone }_{20} \\
\text { Zone }_{8} \\
\text { Zone }_{29} \\
\text { Zone }_{25}\end{array}$ & $\begin{array}{l}1 \text { (Source) } \\
1 \\
1 \\
1 \\
1 \\
1 \\
1 \\
1 \\
2 \\
1\end{array}$ & $\begin{array}{l}\text { Zone }_{1} \\
\text { Zone }_{9} \\
\text { Zone }_{10} \\
\text { Zone }_{11} \\
\text { Zone }_{12} \\
\text { Zone }_{13} \\
\text { Zone }_{14} \\
\text { Zone }_{15} \\
\text { Zone }_{24} \\
\text { Zone }_{23} \\
\text { Zone }_{27} \\
\text { Zone }_{28} \\
\text { Zone }_{8} \\
\text { Zone }_{29} \\
\text { Zone }_{25}\end{array}$ & $\begin{array}{l}1 \text { (Source) } \\
2 \\
1 \\
1 \\
3 \\
2 \\
1 \\
1 \\
1 \text { (UAV) } \\
1 \text { (UAV) } \\
3 \\
2 \\
1 \\
2 \\
1 \text { (Destination) }\end{array}$ \\
\hline \multicolumn{2}{|c|}{ Score $=23.71$} & \multicolumn{2}{|c|}{ Score $=8.39$} & \multicolumn{2}{|c|}{ Score $=6.14$} \\
\hline
\end{tabular}




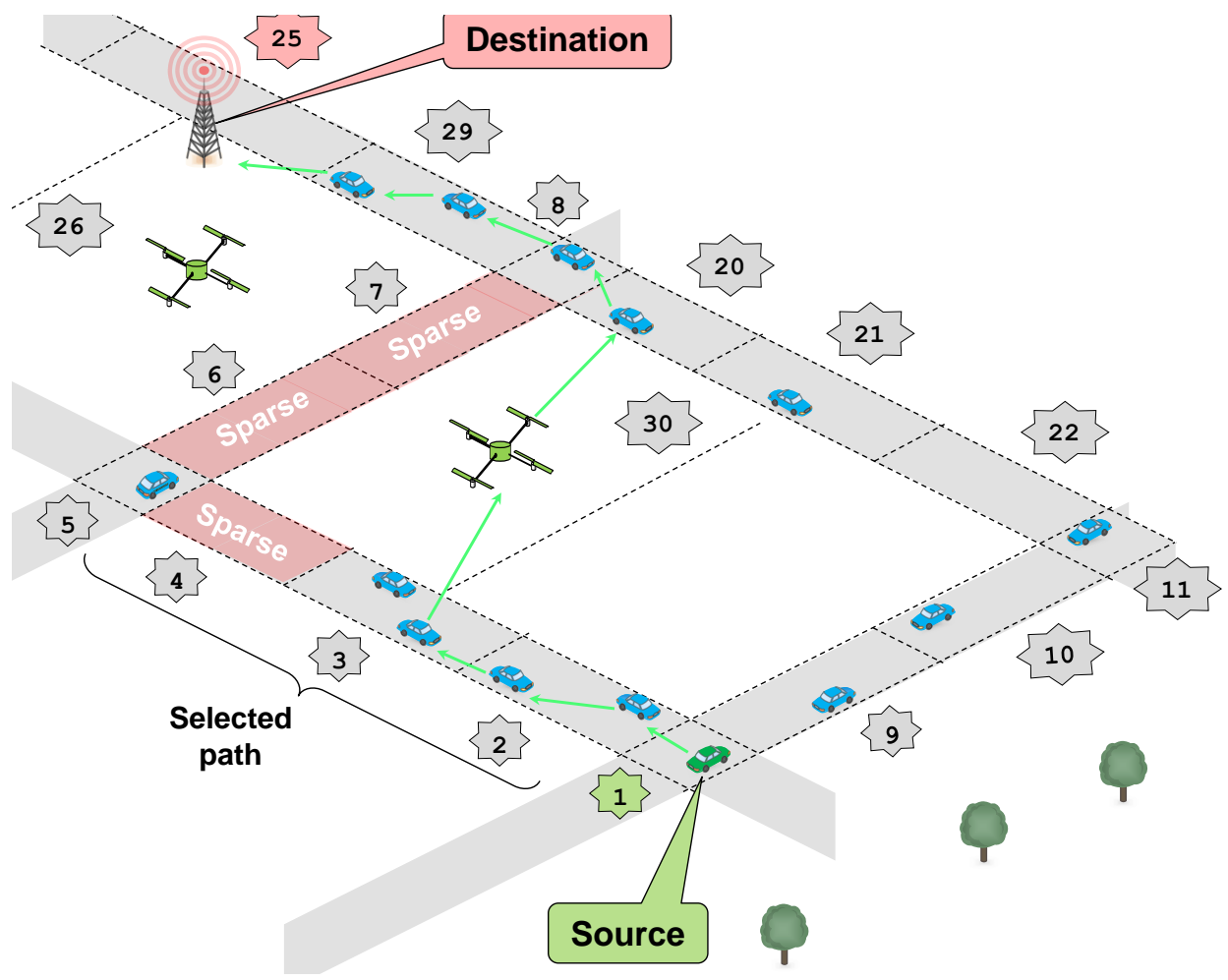

Fig. 10: Path failure recovery.

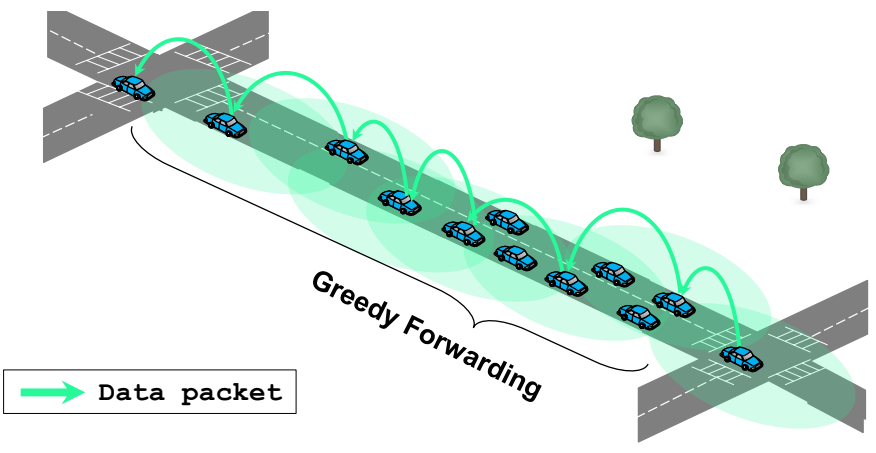

Fig. 11: Data packet's unicast.

To illustrate the data delivery process, we also take the example of Fig. 7. The source vehicle located at Zone $e_{1}$ starts the data delivery to the destination gateway located at Zone 25 . For instance, when a vehicle located at $Z_{o n e}$ intercepts the data packet sent by vehicle $e_{1}$, it firstly analyzes its included header to select the next appropriate zone. This process is repeated by the forwarders at each next zone, where the data packet is automatically transited over them. The destination gateway validates the reception by checking the Destination field included in the header of the data packet.

4) Maintenance process: The maintenance process takes place in the case when the selected path disconnects at a certain time. At the reception of a data packet, the forwarder has the knowledge of the target destination's location and its corresponding zone, which allows using the greedy forwarding for the data delivery. In a general case, if a zone is disconnected in a selected path, the current node has to check its neighboring nodes to find the closest one to the destination. This is carried out using both its knowledge of the whole map and the information included in the header of the data packet (i.e., the knowledge of each zone identifier). If there is no connected zone in its neighboring zones, a route error (RERR) packet is generated and sent back to the source, in order to reestablish a new routing path for the remaining transmissions.

To give a deeper understanding of the maintenance process, we consider the scenario illustrated in Fig. 10 by zooming in Fig. 7. If, for instance, the initially selected $P a t h_{1}$ is broken at its weakest zone Zone $_{4}$. In this case, another zone that is geographically closest to the destination has to be selected to resume the data transmission, i.e., the current forwarder has to check its table of neighbors to know the availability of nodes in the next zones. After this verification, Zone 30 (UAV) is selected to continue the data transmission using the greedy forwarding until the target destination.

According to several conducted simulation scenarios, UAVs always constitute the alternatives when there is a path failure. This adopted recovery strategy significantly minimizes the overhead caused by the dissemination of the different used control packets. In addition, the packet losses and the delivery delay are also reduced due to the minimization of the discovery process at each path failure.

\section{Evaluation}

U2RV is evaluated using the simulation tool NS-2. Realistic movements are generated for both vehicles and UAVs, using two mobility generator tools SUMO [36] and MobiSim [37], respectively. The mobility is based on a real-world urban area as shown in Fig. 12, which knows a flexible and perpetual 
TABLE III: Simulation parameters.

\begin{tabular}{|c|c|c|c|}
\hline \multicolumn{2}{|c|}{ Scenario } & \multicolumn{2}{|c|}{ Technical details } \\
\hline Parameter & Value & Parameter & Value \\
\hline Area & $4000 \mathrm{~m} \times 4000 \mathrm{~m}$ & Vehicle range & $\approx 300 \mathrm{~m}$ \\
\hline Simulation time & $900 \mathrm{~s}$ & UAV range & $\approx 1000 \mathrm{~m}$ \\
\hline Mobility tool & SUMO [36] & MAC Protocol & IEEE $802.11 \mathrm{p}$ \\
\hline Junctions & 47 & Frequency Band & $5.9 \mathrm{GHz}$ \\
\hline Road segments & 100 & Number of packets senders & 50 \\
\hline Vehicles & $80-320$ & Packet size & $1 \mathrm{~KB}$ \\
\hline UAVs & $0-10$ & Channel capacity & $3 \mathrm{Mbps}$ \\
\hline Vehicle speed & $0-50 \mathrm{~km} / \mathrm{h}$ & Ratio of senders & $20 \%$ \\
\hline UAV speed & $0-60 \mathrm{~km} / \mathrm{h}$ & Calculated metrics & $\mathrm{PDR} / \mathrm{EED} / \mathrm{OH} / \mathrm{HOP}$ \\
\hline
\end{tabular}
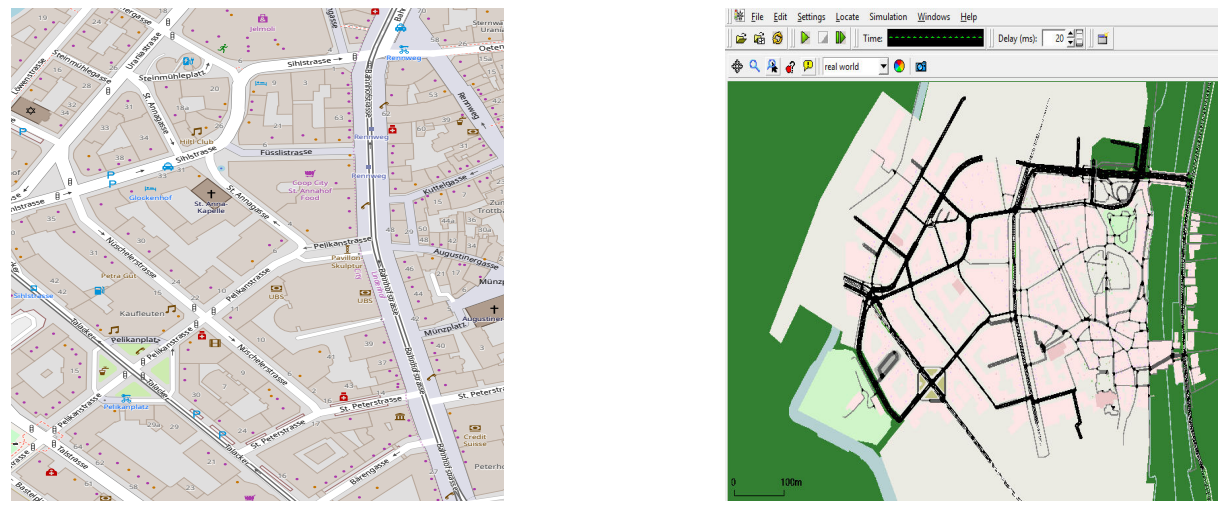

Fig. 12: Map of the simulated area in Zurich, Switzerland ( $\left.47^{\circ} 22^{\prime} 24.7^{\prime \prime} \mathrm{N} 8^{\circ} 32^{\prime} 19.1^{\prime \prime} \mathrm{E}\right)$.

movement of vehicles. For the UAVs, Random Walk (RW) mobility defines the movements of 10 UAVs that can cover the simulated area. The geodata is imported from OpenStreetMap [38] in order to include the road layout in our simulation. This urban map is stretched over $4 \times 4 \mathrm{~km}^{2}$ encompassing nearly 47 intersections, which are compounds of traffic lights where their status change at each 40 (s). We assume that the altitude of all UAVs does not surpass 1000 meters. Numerous evaluation metrics are estimated based on several runs, such as the delivery ratio (PDR), the delivery delay (EED), the average number of hops (HOP), and the control overhead (OH).

In order to study the efficiency of U2RV, its performances are compared to two categories of routing protocols: (i) UAVassisted routing protocols, such as UVAR [11] and CRUV [19], and (ii) VANET routing protocols, such as MURU [25] and AGP [26].The first category allows us to study the impact of using UAVs as relays, whereas the second category allows us to see the effect of using vehicles as relays instead of UAVs. To be more accurate in our simulations, the broadcast of Hello packets is done every 0.1 (s). The list of neighboring nodes of each node is purged after 3 (s) of inactivity. It is worthy to mention that the transmission ranges of vehicles and UAVs are considered as the same when a communication is established between them $(\approx 1000 \mathrm{~m})$.

According to multiple tests in the simulation environment, $80 \%$ is the probability of success for radio transmissions between UAVs. For the hybrid radio transmission (between UAVs and vehicles), the probability of success is set to $70 \%$. The LoS is considered to be guaranteed between vehicles and
UAVs, which are allowed to establish a radio transmission if they are in range of each other. However, non-LoS between vehicles on the ground if they are located at road segments not opposite from each other. To evaluate the simulated protocols in a more realistic way, the initial geographical positions of both vehicles and UAVs are distributed fairly over the network. The mobility of UAVs and vehicles is controlled since their movements are limited only in the simulated area. It is worthy to note that each point of the obtained results represents the mean of 50 simulation runs with $95 \%$ of confidence interval.

The rest of the simulation parameters are outlined in Table III.

\section{A. Packet Delivery Ratio (PDR)}

As a first step, we evaluate the packet delivery ratio (PDR) according to different densities of UAVs and vehicles for all the simulated protocols. The results showed in Fig. 13 depict the proportionality of PDR with the density of vehicles for all the evaluated protocols. In the majority of cases (see Figs. 13(a) and 13(b)), U2RV achieves the highest performance in terms of PDR compared to the other protocols, which can be explained by the adopted technique of the path expiration time prediction. This allows to enhance the data transmission to the destination. Also, this mechanism can ensure a certain reliability of the discovered paths during their maintenance by minimizing the packet losses.

Fig. 13(c) shows the variation of PDR according to the density of UAVs for the UAV-Assisted routing protocols (i.e., U2RV, UVAR, and CRUV). U2RV achieved the highest PDR 


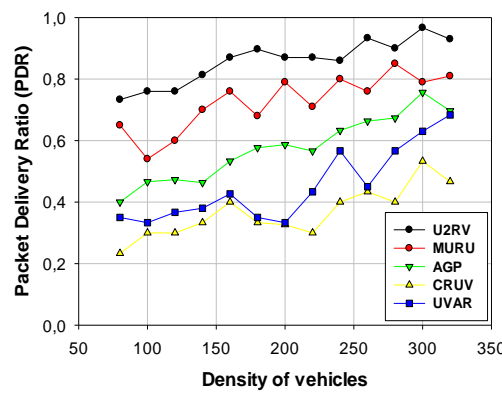

(a) PDR vs. Vehicles $(\mathrm{UAVs}=10)$.

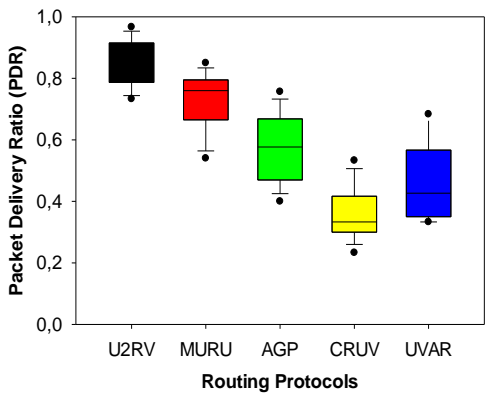

(b) Average packet delivery ratio.

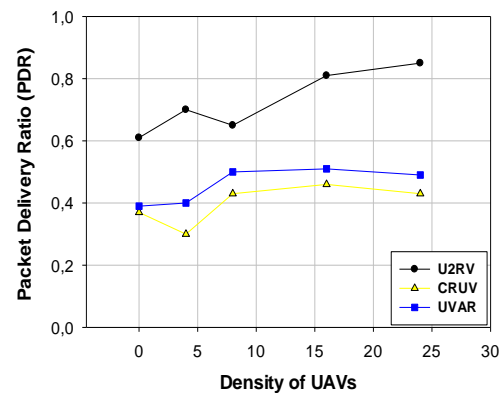

(c) PDR vs. UAVs (Vehicles=200).

Fig. 13: PDR vs. Density of Vehicles and UAVs.

which proves again its efficiency. This is because in the data delivery it relies both on vehicles and UAVs (i.e., paths can be composed of both vehicles and UAVs), which can ensure, at each time, possible paths in the sky and on the ground. However, for the other protocols, they rarely rely on UAVs, and especially if the network is considered as highly dense. It is not the case in the simulated scenario where the number of vehicles is two hundred, which can be considered as a medium density.

\section{B. End-to-End Delay (EED)}

The analysis of the impact of the vehicles' density on the delivery delay performances depicted in Fig. 14. Overall, U2RV performs the data transmission with the lowest delays (c.f., Figs. 14(a) and 14(b)). This is due to both the short periods carried out by the dissemination and the expiration time of paths calculated before the data delivery, which allows to minimize the flooding at each broken path. Nevertheless, MURU does not perform as well as U2RV, thus, it is due to its inefficient maintenance mechanism causing the flooding of the entire network at each link failure. The general causes of delays in the reactive protocols are the flooding process, as well as the selection process that can probably generate additional extra times. Several other causes of additional delays are observed during the simulation. For instance, in the delay tolerant network routing protocols, such as UVAR and CRUV, the store-carry and forward is automatically used at each time when the network is poorly dense generating an important additional delay. The data packets are transmitted based exclusively on the physical movement of vehicles. However, their performances will be quickly recovered as the network becomes progressively connected.

Fig. 14(c) shows the effect of changing the UAV density on the delivery delay of the UAV-assisted routing protocols. Our approach obtains the best results compared with other protocols. This is essentially due to the efficient discovery process, which involves vehicles and UAVs as well. However, it is not the case of CRUV and UVAR, which use UAVs only for the data delivery in certain cases, and in the majority of cases as central points in the selection processes (collectors). In addition, their excessive use of the store-carry-and-forward can be also a disadvantageous factor increasing the delay.

\section{Average Number of Hops (HOP)}

Fig. 15 depicts the variation of the average number of hops according to the vehicle density for the evaluated protocols. It is clearly observed that the number of hops of UVAR and CRUV essentially caused by two factors: (i) the long distances between the communicating nodes, and (ii) the selection of the densest road segments and at a certain time without taking into consideration the distances separating each pair of communicating nodes (c.f., Figs. 15(a) and 15(b)). However, for the reactive protocols (U2RV and MURU), initially (low densities 80-180) they have frequent failures due to the highly dynamic

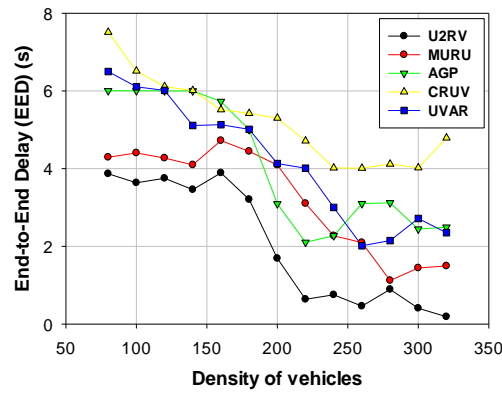

(a) EED vs. Vehicles (UAVs=10).

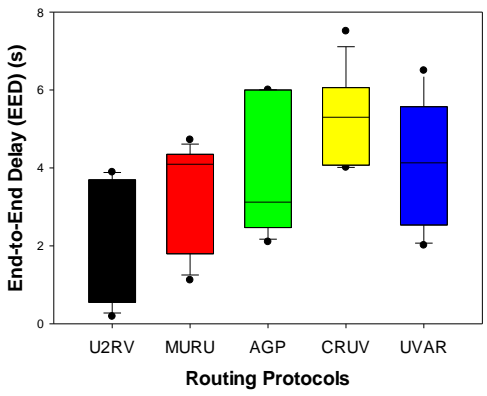

(b) Average end-to-end delay.

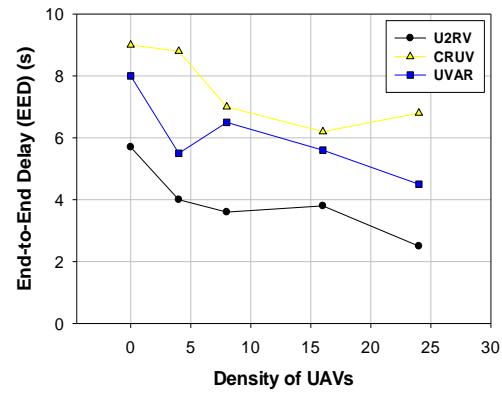

(c) EED vs. UAVs (Vehicles=200).

Fig. 14: EED vs. Density of Vehicles and UAVs. 


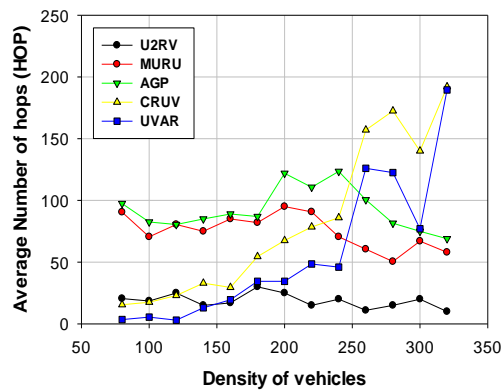

(a) HOP vs. Vehicles $(\mathrm{UAVs}=10)$.

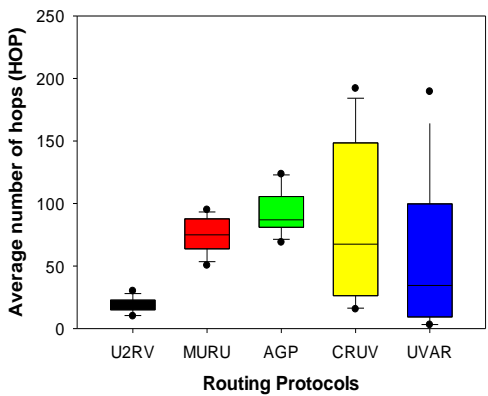

(b) Average number of hops.

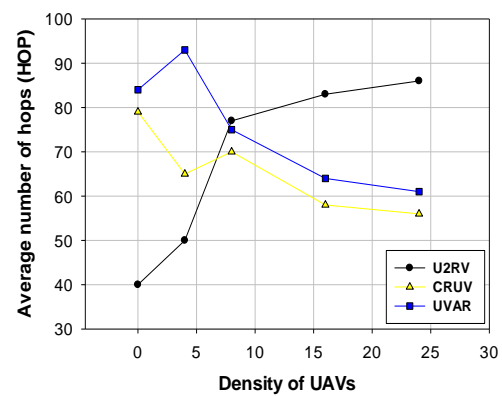

(c) HOP vs. UAVs (Vehicles=200).

Fig. 15: HOP vs. Density of Vehicles and UAVs.

mobility of nodes. This imposes to the reactive protocols to reinitiate the discovery process for each data request increasing at each time the number of hops for a requested data packet. In a general case, we can say that the number of hops is approximately stable except for the higher densities (240-320). In U2RV, we distinguished a slight decrease caused by the use of the greedy forwarding, and especially when UAVs are used as relays. This can minimize considerably the number of hops. The same observation is distinguished for MURU, where the number of hops decreases for the higher densities. However, due to occasional disconnections on the network, MURU can re-initialize the discovery process.

Fig. 15(c) clearly illustrates that the number of hops of U2RV is significantly higher than CRUV and UVAR for the 8,16 and 24 UAV densities. This can be explained by the increase of UAVs, the paths which are composed of both vehicles and UAVs will be increased, and therefore, the number of hops will increase. However, for UVAR and CRUV, the number of hops decreases as the density of UAVs is raised, which is mainly due to the increase of the UAVs coverage in the simulated area. This can facilitate the frequent use of vehicles and UAVs for data delivery, which significantly reduces the number of hops.

\section{Control Overhead $(\mathrm{OH})$}

The curves and box plots presented in Figs. 16(a) and 16(b), respectively, show the overhead for each density variation. It can be shown that the overhead of AGP, MURU, and U2RV is important compared to CRUV and UVAR. Nevertheless, U2RV achieves the lowest overhead compared to the reactive protocols, which can be explained by the use of the prediction of the path expiration allowing it to have long duration connectivity. This allows to reduce the flooding at each broken path. As for UVAR and CRUV, they generate less overhead packets because they use only the periodical exchange of the Hello packets between the nodes.

In Fig. 16(c), the results clearly show that UVAR and CRUV produce less overhead than the reactive protocols, which is essentially caused by the extra generated control packets. The more the density of UAVs, the more will be the number of generated control packets during the flooding process. However, as for UVAR and CRUV, the UAVs are both used as traffic density information collectors and as relays. Consequently, their control overheads are very low and stay approximately constant during all the simulations. UVAR has more control overhead than CRUV, which is explained by the important exchange of Hello packets between UAVs and all vehicles. However, it is not the case of CRUV, in which Hello packets are only exchanged between vehicles located at the intersections and UAVs in order to calculate the scores of each road segment. Furthermore, in CRUV, the UAVs are not involved in the selection of the road segments.

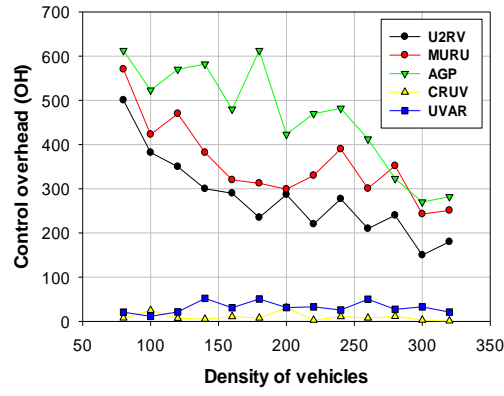

(a) $\mathrm{OH}$ vs. Vehicles $(\mathrm{UAVs}=10)$.

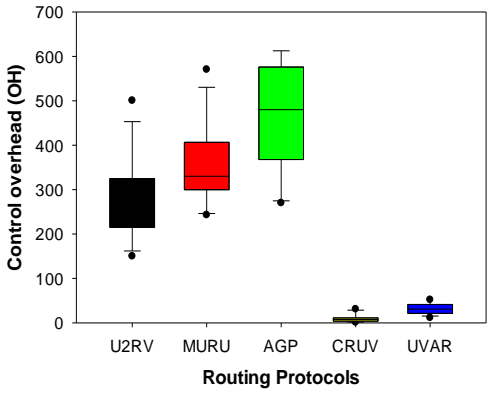

(b) Average control overhead.

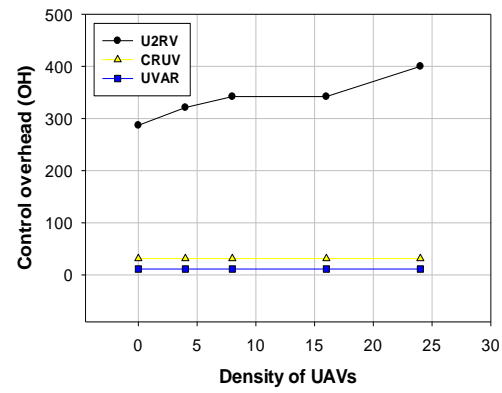

(c) $\mathrm{OH}$ vs. UAVs (Vehicles=200).

Fig. 16: OH vs. Density of Vehicles and UAVs. 


\section{CONCLUSION}

A novel reactive routing protocol has been proposed. This scheme exploits a multi-criteria selection of paths which allows benefiting from the advantages of well-stable and regulated paths towards fixed or mobile nodes. Several applications requiring a minimum of packet losses can be supported by this routing scheme. By including a prediction method, this scheme is able to accurately predict the expiration time at each discovery process initialized by a requesting node. In addition to vehicles, UAVs are dispersed in the sky in which can also belong to the discovered paths, and consequently, participate in the data delivery and act as relays. Also, UAVs have demonstrated their crucial role in the majority of time during the maintenance process due to their localization, which allows vehicles to find the appropriate support when a path will be disconnected. Performance results are presented, which clearly justify our claims for the use of this heterogeneous wireless communication between UAVs and vehicles in terms of delay of transmission and throughput. We have noticed that the data delivery in our approach can be done in a near-optimal delivery delay with a minimized overhead and reduced number of hops. However, it is not the case of the routing protocols proposed in the same category. The detection of path failures by placing UAVs in the right places and the adaptation to different environments and situations, could become a portion of future work. Moreover, we plan to integrate a security component including cryptography schemes in order to deal with some attacks, such as: (i) attack coordination which can inject excessively falsified RREQ packets to cause a broadcast storm and thus denial of service (DoS) can occur, (ii) nodes behavior which consists altering control packet, especially during the route discovery, which is crucial for routing decisions, and (iii) passive attack which can occur due to limited resources in the network, such as bandwidth and processing capabilities.

\section{REFERENCES}

[1] C. Bouras, A. Gkamas, V. Kapoulas, D. Politaki, and E. Tsanai, "Video transmission in mobile ad hoc networks using multiple interfaces and multiple channels," International Journal of Communication Systems, vol. 30, no. 8, p. e3172, 2017.

[2] S. H. H. Nazhad, M. Shojafar, S. Shamshirband, and M. Conti, "An efficient routing protocol for the QoS support of large-scale MANETs," International Journal of Communication Systems, vol. 31, no. 1, p. e3384, 2018.

[3] S. Benkerdagh and C. Duvallet, "Cluster-based emergency message dissemination strategy for VANET using V2V communication," International Journal of Communication Systems, p. e3897.

[4] L. Alouache, N. Nguyen, M. Aliouat, and R. Chelouah, "Survey on IoV routing protocols: Security and network architecture," International Journal of Communication Systems, vol. 32, no. 2, p. e3849, 2019.

[5] O. S. Oubbati, A. Lakas, F. Zhou, M. Güneş, N. Lagraa, and M. B. Yagoubi, "Intelligent UAV-Assisted Routing Protocol for Urban VANETs," Computer communications, vol. 107, pp. 93-111, 2017.

[6] M. Chahal and S. Harit, "A stable and reliable data dissemination scheme based on intelligent forwarding in VANETs," International Journal of Communication Systems, vol. 32, no. 3, p. e3869, 2019.

[7] A. K. Ligo, J. M. Peha, and J. Barros, "Is It Cost-Effective to Share Roadside Infrastructure for Internet Access?" in Proceedings of the IEEE 85th Vehicular Technology Conference (VTC Spring). IEEE, 2017, pp. $1-7$.

[8] A. K. Ligo and J. M. Peha, "Cost-Effectiveness of Sharing Roadside Infrastructure for Internet of Vehicles," IEEE Transactions on Intelligent Transportation Systems, vol. 19, no. 7, pp. 2362-2372, 2018.
[9] A. Bazzi and A. Zanella, "Position based routing in crowd sensing vehicular networks," Ad Hoc Networks, vol. 36, pp. 409-424, 2016.

[10] T. Darwish, K. A. Bakar, and A. Hashim, "Green geographical routing in vehicular ad hoc networks: Advances and challenges," Computers \& Electrical Engineering, vol. 64, pp. 436-449, 2017.

[11] O. S. Oubbati, A. Lakas, N. Lagraa, and M. B. Yagoubi, "UVAR: An intersection UAV-assisted VANET routing protocol," in Proceedings of the Wireless Communications and Networking Conference (WCNC). IEEE, 2016, pp. 1-6.

[12] K. E. Suleiman and O. Basir, "Adaptive incentive-compatible routing in VANETs for highway applications," in Proceedings of the 8th IEEE Annual Information Technology, Electronics and Mobile Communication Conference (IEMCON). IEEE, 2017, pp. 670-679.

[13] J. He, L. Cai, J. Pan, and P. Cheng, "Delay analysis and routing for two-dimensional VANETs using carry-and-forward mechanism," IEEE Transactions on Mobile Computing, vol. 16, no. 7, pp. 1830-1841, 2017.

[14] M. T. Garrosi, X. Xiang, and M. Noroozi, "Loop-Free Enhanced Intersection-Based Perimeter Geo-Routing with Carry-and-Forward for Urban VANETs," in Proceedings of the IEEE 86th Vehicular Technology Conference (VTC-Fall). IEEE, 2017, pp. 1-7.

[15] T. Saeed, Y. Mylonas, A. Pitsillides, V. Papadopoulou, and M. Lestas, "Modeling Probabilistic Flooding in VANETs for Optimal Rebroadcast Probabilities," IEEE Transactions on Intelligent Transportation Systems, vol. 20, no. 2, pp. 556-570, 2018.

[16] O. S. Oubbati, A. Lakas, M. Güneş, F. Zhou, and M. B. Yagoubi, "UAVassisted reactive routing for urban VANETs," in Proceedings of the ACM Symposium on Applied Computing (SAC). ACM, 2017, pp. 651-653.

[17] P. Fazio, F. De Rango, and C. Sottile, "A predictive cross-layered interference management in a multichannel MAC with reactive routing in VANET," IEEE Transactions on Mobile Computing, vol. 15, no. 8, pp. 1850-1862, 2016.

[18] P. Sewalkar and J. Seitz, "Vehicle-to-pedestrian communication for vulnerable road users: survey, design considerations, and challenges," Sensors, vol. 19, no. 2, p. 358, 2019.

[19] O. S. Oubbati, A. Lakas, N. Lagraa, and M. B. Yagoubi, "CRUV: Connectivity-based traffic density aware routing using UAVs for VANETs," in Proceedings of International Conference on Connected Vehicles and Expo (ICCVE), 2015, pp. 68-73.

[20] O. S. Oubbati, A. Lakas, F. Zhou, M. Güneş, and M. B. Yagoubi, "A survey on position-based routing protocols for Flying Ad hoc Networks (FANETs)," Vehicular Communications, vol. 10, pp. 29-56, 2017.

[21] O. S. Oubbati, N. Chaib, A. Lakas, and S. Bitam, "On-Demand Routing for Urban VANETs using Cooperating UAVs," in Proceedings of the 7th International Conference on Smart Communications in Network Technologies (SaCoNeT). IEEE, 2018, pp. 108-113.

[22] J. Zhu, C. Huang, X. Fan, and B. Fu, "An Efficient Distributed Randomized Data Replication Algorithm in VANETs," in Proceedings of the International Conference on Wireless Algorithms, Systems, and Applications. Springer, 2017, pp. 369-380.

[23] T. Taleb, E. Sakhaee, A. Jamalipour, K. Hashimoto, N. Kato, and Y. Nemoto, "A stable routing protocol to support ITS services in VANET networks," IEEE Transactions on Vehicular Technology, vol. 56, no. 6, pp. 3337-3347, 2007.

[24] S. Zaidi, S. Bitam, and A. Mellouk, "Hybrid error recovery protocol for video streaming in vehicle ad hoc networks," Vehicular communications, vol. 12, pp. 110-126, 2018.

[25] Z. Mo, H. Zhu, K. Makki, and N. Pissinou, "MURU: A multi-hop routing protocol for urban vehicular ad hoc networks," in Proceedings of the Third Annual International Conference on Mobile And Ubiquitous Systems: Networking \& Services. IEEE, 2006, pp. 1-8.

[26] S. Yan, X.-y. JIN, and S.-z. CHEN, "AGP: an anchor-geography based routing protocol with mobility prediction for VANET in city scenarios," The Journal of China Universities of Posts and Telecommunications, vol. 18, pp. 112-117, 2011.

[27] W. Sun, H. Yamaguchi, K. Yukimasa, and S. Kusumoto, "GVGrid: A QoS routing protocol for vehicular ad hoc networks," in Proceedings of the 14th IEEE International Workshop on Quality of Service, 2006, pp. $130-139$.

[28] R. Shirani, M. St-Hilaire, T. Kunz, Y. Zhou, J. Li, and L. Lamont, "On the delay of reactive-greedy-reactive routing in unmanned aeronautical ad-hoc networks," Procedia Computer Science, vol. 10, pp. 535-542, 2012.

[29] E. Sakhaee and A. Jamalipour, "A new stable clustering scheme for pseudo-linear highly mobile ad hoc networks," in Proceedings of IEEE Global Telecommunications Conference (GLOBECOM'07),. IEEE, 2007, pp. 1169-1173. 
[30] M. Iordanakis, D. Yannis, K. Karras, G. Bogdos, G. Dilintas, M. Amirfeiz, G. Colangelo, and S. Baiotti, "Ad-hoc routing protocol for aeronautical mobile ad-hoc networks," in Proceeding of the Fifth International Symposium on Communication Systems, Networks and Digital Signal Processing (CSNDSP). Citeseer, 2006.

[31] C. Perkins, E. Belding-Royer, and S. Das, "Ad hoc on-demand distance vector (aodv) routing," Tech. Rep., 2003.

[32] H. Menouar, I. Guvenc, K. Akkaya, A. S. Uluagac, A. Kadri, and A. Tuncer, "UAV-enabled intelligent transportation systems for the smart city: Applications and challenges," IEEE Communications Magazine, vol. 55 , no. 3, pp. 22-28, 2017.

[33] L. Gupta, R. Jain, and G. Vaszkun, "Survey of important issues in UAV communication networks," IEEE Communications Surveys \& Tutorials, vol. 18, no. 2, pp. 1123-1152, 2016.

[34] W. Su, S.-J. Lee, and M. Gerla, "Mobility prediction and routing in ad hoc wireless networks," International Journal of Network Management, vol. 11, no. 1, pp. 3-30, 2001.

[35] K. Liu, J. Zhang, and T. Zhang, "The clustering algorithm of UAV networking in near-space," in Proceedings of the 8th International Symposium on Antennas, Propagation and EM Theory (ISAPE). IEEE, 2008, pp. 1550-1553.

[36] M. Behrisch, L. Bieker, J. Erdmann, and D. Krajzewicz, "SUMOsimulation of urban mobility: an overview," in Proceedings of the Third International Conference on Advances in System Simulation (SIMUL 2011), 2011

[37] S. M. Mousavi, H. R. Rabiee, M. Moshref, and A. Dabirmoghaddam, "Mobisim: A framework for simulation of mobility models in mobile ad-hoc networks," in Proceedings of the Third IEEE International Conference on Wireless and Mobile Computing, Networking and Communications (WiMOB), 2007, pp. 82-82.

[38] M. Haklay and P. Weber, "Openstreetmap: User-generated street maps," IEEE Pervasive Computing, vol. 7, no. 4, pp. 12-18, 2008.

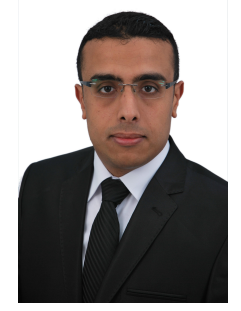

Omar Sami Oubbati is an Associate Professor at the Electronics department, University of Laghouat, Algeria and a Research Assistant in the Computer Science and Mathematics Lab (LIM) at the same university. He received his degree of Engineer (2010), M.Sc. in Computer Engineering (2011), M.Sc. degree (2014), and a PhD in Computer Science (2018). From Oct. 2016 to Oct. 2017, he was a Visiting Student with the Laboratory of Computer Science, University of Avignon, France. His main research interests are in Flying and Vehicular ad hoc networks, Visible light communications, Energy efficiency and Internet of Things (IoT). He is a reviewer in many international journals and a TPC member in many international conferences. He is a member of the IEEE and IEEE Communications Society.

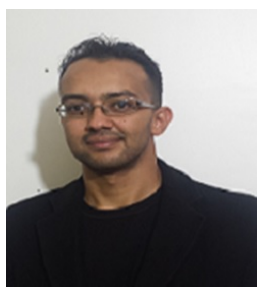

Noureddine Chaib received his $\mathrm{PhD}$ degree in computer science from University of Laghouat and serves as treasurer for IEEE Algeria Section. He is currently an associate professor in the Computer Science Department, the Chief Information Officer and Vice chancellor of University of Laghouat. He is a member of the Computer Science and Mathematics Lab (LIM) and a member of IEEE Vehicular Technology Society. He received the degree of engineering in computer science from University of Laghouat, in 2007, and the degree of Master of engineering in computer science from the University of Batna, in 2011. His research interests include Security, Privacy, Mobile and Vehicular networks and other networking topics.

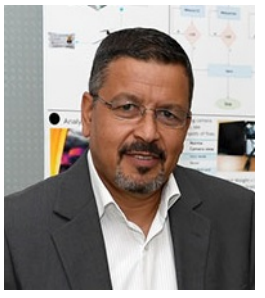

Aberrahmane Lakas received his MS (1990) and $\mathrm{PhD}$ (1996) in Computer Systems from the University of Paris VI, Paris, France. He joined the College of Information Technology, UAE University in 2003. $\mathrm{He}$ is teaching various courses on computer networks and network security. Dr. Lakas had many years of industrial experience holding various technical positions in telecommunication companies, such as Netrake (Plano, Texas, 2002), Nortel (Ottawa, 2000) and Newbridge (Ottawa, 1998). Dr. Lakas is in the editorial board of Journal of Communications (Actapress), and Journal of Computer Systems, Networks, and Communications (Hindawi).

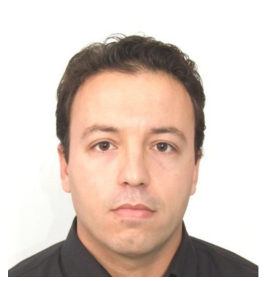

Salim Bitam received the State Engineer degree in computer science from Mentouri University, Constantine, Algeria, in 1999 and the Magister and Doctorate in science degrees in computer science from Mohamed Khider University, Biskra, Algeria, in 2002 and 2011, respectively. In December 2002, he has been an assistant professor and since July 2018 he is a full professor in computer science department and a senior member of Laboratory of Expert Systems, Imagery and their Applications in Engineering (LESIA) in University of Biskra. His main research interests are cloud computing, mobile ad hoc networks, vehicular ad hoc networks, wireless sensor networks and bio-inspired methods for optimization. Prof. Salim Bitam has to his credit more than 40 publications in journals, books and conferences, in which he has received two best paper awards. He has served as an editorial member and a reviewer of several journals, such as Elsevier and Springer and on the technical program committees of several international conferences.

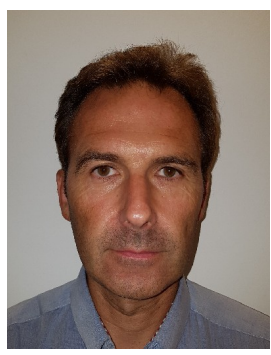

Pascal Lorenz received his M.Sc. (1990) and Ph.D. (1994) from the University of Nancy, France. Between 1990 and 1995 he was a research engineer at WorldFIP Europe and at Alcatel-Alsthom. He is a professor at the University of Haute-Alsace, France, since 1995. His research interests include QoS, wireless networks and high-speed networks. He is the author/co-author of 3 books, 3 patents and 200 international publications in refereed journals and conferences. He was Technical Editor of the IEEE Communications Magazine Editorial Board (20002006), IEEE Networks Magazine since 2015, IEEE Transactions on Vehicular Technology since 2017, Chair of IEEE ComSoc France (2014-2018), Financial chair of IEEE France (2017-2019), Chair of Vertical Issues in Communication Systems Technical Committee Cluster (2008-2009), Chair of the Communications Systems Integration and Modeling Technical Committee (2003-2009), Chair of the Communications Software Technical Committee (2008-2010) and Chair of the Technical Committee on Information Infrastructure and Networking (2016-2017). He has served as Co-Program Chair of IEEE WCNC'2012 and ICC'2004, Executive Vice-Chair of ICC'2017, Panel sessions co-chair for Globecom'16, tutorial chair of VTC'2013 Spring and WCNC'2010, track chair of PIMRC'2012 and WCNC'2014, symposium Co-Chair at Globecom 2007-2011, ICC 2008-2010, ICC'2014 and '2016. He has served as CoGuest Editor for special issues of IEEE Communications Magazine, Networks Magazine, Wireless Communications Magazine, Telecommunications Systems and LNCS. He is associate Editor for International Journal of Communication Systems (IJCS-Wiley), Journal on Security and Communication Networks (SCN-Wiley) and International Journal of Business Data Communications and Networking, Journal of Network and Computer Applications (JNCAElsevier). He is senior member of the IEEE, IARIA fellow and member of many international program committees. He has organized many conferences, chaired several technical sessions and gave tutorials at major international conferences. He was IEEE ComSoc Distinguished Lecturer Tour during 20132014. 